\title{
Photon management with lanthanides
}

\author{
Andries Meijerink *, René Wegh, Peter Vergeer, Thijs Vlugt \\ Debye Institute, Department of Chemistry, Utrecht University, Princetonplein 5, 3584 CC Utrecht, The Netherlands
}

Available online 8 November 2005

\begin{abstract}
Efficient conversion of photons from high energy radiation (e.g. ultraviolet or X-rays) to lower energies (visible) has been optimized by using luminescent materials based on the optical properties of lanthanide ions. Presently, luminescent materials with efficiencies close to the theoretical maximum are applied in e.g. fluorescent tubes, X-ray imaging and color television. Contrary to the mature status of luminescent materials in these fields, areas requiring new luminescent materials are emerging. There is great challenge in research on upand downconversion materials and lanthanide ions are the prime candidates to achieve efficient materials. Here downconversion processes will be discussed for VUV phosphors with $\mathrm{Pr}^{3+}$. The efficiency of resonant energy transfer of the ${ }^{1} \mathrm{~S}_{0}{ }^{-1} \mathrm{I}_{6}$ energy from $\operatorname{Pr}^{3+}$ to $\mathrm{Eu}^{3+}$ and $\mathrm{Mn}^{2+}$ is investigated. The aim is to convert the $405 \mathrm{~nm}$ photon of the first step of the well-known cascade emission of $\mathrm{Pr}^{3+}$ into a more useful visible photon. For co-doping with $\mathrm{Eu}^{3+}$ it is observed that the $\mathrm{Pr}^{3+}$ emission is quenched, most probably through a metal-to-metal charge-transfer state. The energy transfer from $\mathrm{Pr}^{3+}$ to $\mathrm{Mn}^{2+}$ is found to be inefficient.

An alternative for downconversion through resonant energy transfer is the non-resonant process of cooperative sensitization. For the $\mathrm{Tb}-\mathrm{Yb}$ couple efficient cooperative energy transfer from the ${ }^{5} \mathrm{D}_{4}$ level of $\mathrm{Tb}^{3+}$ to two $\mathrm{Yb}^{3+}$ neighbors is observed with a transfer rate of $0.26 \mathrm{~ms}^{-1}$. This corresponds to an upper limit of $188 \%$ for the conversion efficiency of visible $(490 \mathrm{~nm})$ photons to infrared $(\sim 1000 \mathrm{~nm})$ photons.
\end{abstract}

(c) 2005 Elsevier B.V. All rights reserved.

\section{Introduction}

The lanthanides occupy a special place in the periodic table of elements. They are situated at the bottom of the periodic table, one row above the actinides. The word lanthanide has a Greek origin (" $\lambda \alpha v \theta \alpha \nu \varepsilon v v$ ") which means "to lie hidden". This may seem appropriate in view of the position of the lanthanides in the periodic table and the fact that it took more than a century to separate and discover all the lanthanides. Nevertheless, at present lanthanides are prominently present in a wide range of products related to e.g. the catalytic, magnetic and optical properties of the lanthanide ions. Especially in optical applications the lanthanides have become (literally) visible in the past decades. The applications of lanthanide ions in the field of optical materials is related to the unique energy level diagrams of

\footnotetext{
* Corresponding author. Tel.: +3130253 2202; fax: +31302532403.

E-mail address: a.meijerink@phys.uu.nl (A. Meijerink).
}

the lanthanides which are known as the Dieke diagram. The rich energy level structure make that lanthanide ions are perfect "photon managers" that can be used to efficiently convert radiation into light of any desired wavelength [1]. In the past four decades the use of lanthanide ions as photon managers has rapidly increased.

The first commercial luminescent material based on lanthanide luminescence was $\mathrm{YVO}_{4}: \mathrm{Eu}^{3+}$. This material was discovered in the early 1960 s and found to efficiently convert the energy of high energy electrons into visible (red) light in a color television. The high luminescence efficiency triggered the application of lanthanide luminescence in other areas, for example in luminescent materials for fluorescent tubes and X-ray imaging. The fluorescence tube is based on a mercury discharge and research has resulted in luminescent materials (phosphors) for the conversion of $254 \mathrm{~nm}$ ultraviolet (UV) radiation into visible light. With the introduction of lanthanide-based luminescent materials, the quantum efficiency for the conversion of $254 \mathrm{~nm}$ UV radiation into red, green or blue light has reached 
the limit of close to $100 \%$. Further research on lamp phosphors is mainly aimed at cost price reduction and marginal improvements of the efficiency or stability. Due to the mature status of the product, research on luminescent materials for mercury discharge fluorescent tubes has strongly decreased in the past decades. The same is true for luminescent materials used in cathode ray tubes and $\mathrm{X}$-ray imaging. The efficiency of these materials is close to the theoretical maximum and research on new luminescent materials for these applications has decreased due to the ideal photon management qualities of lanthanides.

On the other hand, new areas of research have emerged in the past decades. Extensive research is conducted for e.g. (upconversion) lasers based on lanthanides, lanthanide doped fiber amplifiers for telecommunication, 3-D television with lanthanide doped crystals or glasses, scintillators and optical switches [1,2]. Lanthanide doped photonic materials research has become a prominent area of research. Two specific areas in the field that have recently emerged are the search for more efficient and stable luminescent materials for the conversion of high energy UV (vacuum ultraviolet, VUV) radiation into visible light (for xenon-based fluorescent tubes and plasma display panels) and the conversion of low energy UV or blue light (for white light $\mathrm{GaN}$ diodes). The search for new luminescent materials for the conversion of vacuum ultraviolet radiation from a xenon discharge (around $172 \mathrm{~nm}$ ) into visible light involves research on finding ways for the generation of two visible photons for a single VUV photon $[3,4]$. This so-called quantum cutting process is possible using the energy level structure of lanthanide ions. After an introduction into the VUV spectroscopy of lanthanides, this paper will discuss recent developments in the field of quantum cutting. Subsequently, efficient quantum cutting by cooperative energy transfer is demonstrated for the $\mathrm{Tb}-\mathrm{Yb}$ couple converting one visible photon into two infrared photons. The visible to infrared downconversion with quantum yields close to $200 \%$ may be used to increase the efficiency of solar cells.

\section{Experimental}

\subsection{Sample preparation}

In all experiments measurements are performed on microcrystalline powders synthesized by common solid state synthesis. Fluorides were prepared by mixing starting fluorides in stoichiometric ratios, adding $\sim 2 \mathrm{~g}$ of ammoniumfluoride and firing the intimate mixture in a nitrogen atmosphere at typically $600^{\circ} \mathrm{C}$. Powder samples of $\mathrm{Yb}_{x} \mathrm{Y}_{0.99-x} \mathrm{~Tb}_{0.01} \mathrm{PO}_{4}$ were prepared from stock solutions of $\mathrm{Yb}^{3+}$ and $\mathrm{Y}^{3+}$, both containing $1 \%$ of $\mathrm{Tb}^{3+}$, prepared by dissolving rare earth (RE) oxides in concentrated hydrochloric acid. The rare earth ions in the mixed solution were precipitated by adding a solution with an excess of oxalic acid. The precipitate was washed thoroughly to eliminate chloride ions. Heating the compound to $1050{ }^{\circ} \mathrm{C}$ under an oxygen atmosphere converted the oxalates to oxides. The powder obtained was mixed with diammoniumphosphate (10 mol\% excess) and fired again at $1350{ }^{\circ} \mathrm{C}$ under a $1: 3$ $\mathrm{H}_{2}: \mathrm{N}_{2}$ atmosphere. All samples were checked for phase purity using powder-XRD and found to be single-phase.

\subsection{Optical measurements}

Emission and excitation measurements were performed using a SPEX DM3000F spectrofluorometer with a $450 \mathrm{~W}$ Xe-lamp as excitation source. The excitation light was dispersed by a double-grating $0.220 \mathrm{~m}$ SPEX 1680monochromator $(1200 \mathrm{l} / \mathrm{mm})$ blazed at $300 \mathrm{~nm}$. The light emitted by the sample was focussed on a fiber guiding the light to a monochromator where the emission light was dispersed by a $150 \mathrm{l} / \mathrm{mm}$ grating (Scientific Spectra Pro) blazed at $500 \mathrm{~nm}$ with $0.300 \mathrm{~m}$ focal length and detected using a Princeton Instruments 300i CCD camera. VUV spectroscopy was done at a SPEX DM3000F spectrofluoremeter adapted for the VUV and at the HIGITI beamline of the DESY synchrotron in Hamburg. Time-resolved measurements were performed using a Lamba Physic LPD3000 tunable dye laser filled with a Coumarine 307 dye solution. It is pumped by a Lambda Physic LPX100 excimer $(\mathrm{XeCl})$ laser. The typical pulse width of this setup is $\sim 20 \mathrm{~ns}$. The emission was detected using a Hamamatsu R928 PMT and monitored as a function of time using a Tektronix 2440 digital oscilloscope.

\section{Vacuum ultraviolet spectroscopy and quantum cutting}

The vacuum ultraviolet region of the electromagnetic spectrum covers the region between 50 and $200 \mathrm{~nm}$ (200000-50000 $\mathrm{cm}^{-1}$ ). Research on the VUV energy levels of lanthanides has been very limited until recently. Extensive research in this area was triggered by the need of new phosphors for VUV excitation for mercury free fluorescent tubes and plasma display panels. In these devices the VUV radiation generated in a Xe discharge (around $172 \mathrm{~nm}$ ) needs to be converted into visible light. For the intraconfigurational $4 \mathrm{f}^{n}$ levels the VUV research has resulted in the extension of the Dieke diagrams into the VUV [5]. For the $4 \mathrm{f}^{n-1} 5 \mathrm{~d}$ levels high resolution excitation VUV spectra were measured and a model was developed explaining the $4 \mathrm{f}^{n-1} 5 \mathrm{~d}$ energy level structure [6]. In addition to the increased fundamental understanding of the VUV energy levels of lanthanides, an efficient process for the conversion of one VUV photon into two visible photons was found in gadolinium fluorides doped with $\mathrm{Eu}^{3+}$. A two-step resonant energy-transfer process starting from the ${ }^{6} \mathrm{G}$ level of $\mathrm{Gd}^{3+}$ results in the emission of two visible (red) photons emitted by two $\mathrm{Eu}^{3+}$ ions [3]. An alternative quantum cutting process involves a cascade emission from the ${ }^{1} \mathrm{~S}_{0}$ level of $\operatorname{Pr}^{3+}[7,8]$. Albeit efficient, the photon emitted in the first step has a wavelength around $405 \mathrm{~nm}$ which is not favorable for lighting applications. In order to convert this $405 \mathrm{~nm}$ photon into a more useful wavelength, 
two couples of luminescent ions were investigated, viz. $\mathrm{Pr}-$ $\mathrm{Eu}$ and $\mathrm{Pr}-\mathrm{Mn}$.

For the $\mathrm{Pr}-\mathrm{Eu}$ couple efficient energy transfer through the cross-relaxation process $\operatorname{Pr}^{3+}\left({ }^{1} \mathrm{~S}_{0} \rightarrow{ }^{1} \mathrm{I}_{6}\right)$ and $\mathrm{Eu}^{3+}\left({ }^{7} \mathrm{~F}_{0,1} \rightarrow{ }^{5} \mathrm{D}_{3},{ }^{5} \mathrm{~L}_{6}\right)$ is expected. Zachau and coworkers tried to transfer the energy of the $\operatorname{Pr}^{3+1} \mathrm{~S}_{0} \rightarrow{ }^{1} \mathrm{I}_{6}$ transition to the $\mathrm{Eu}^{3+}$ ion [9]. Despite the presence of good spectral overlap between the $\mathrm{Pr}^{3+}$ emission and $\mathrm{Eu}^{3+}$ absorption lines no $\mathrm{Eu}^{3+}$ emission was observed upon excitation of $\mathrm{Pr}^{3+}$. Based on these observations it was concluded that the expected energy transfer does not occur. This is remarkable, since an important condition for energy transfer, the presence of resonance between the $\operatorname{Pr}^{3+}{ }^{3} \mathrm{~S}_{0} \rightarrow{ }^{1} \mathrm{I}_{6}$ transition and absorptions of the $\mathrm{Eu}^{3+}$ ion is fulfilled. In this case energy transfer can occur through exchange interaction or multipole-multipole interaction.

Calculations of the energy-transfer rate assuming dipole-dipole interaction show that energy transfer between nearest-neighbor $\operatorname{Pr}^{3+1} \mathrm{~S}_{0} \rightarrow{ }^{1} \mathrm{I}_{6}$ and $\mathrm{Eu}^{3+7} \mathrm{~F}_{0,1} \rightarrow{ }^{5} \mathrm{D}_{3},{ }^{5} \mathrm{~L}_{6}$ has a similar probability as radiative decay from the $\operatorname{Pr}^{3+1} \mathrm{~S}_{0}$ level. As a result, energy transfer and subsequent $\mathrm{Eu}^{3+}$ emission should occur. To investigate the observed absence of $\mathrm{Eu}^{3+}$ emission upon excitation in the ${ }^{1} \mathrm{~S}_{0}$ level of $\operatorname{Pr}^{3+}$ luminescence spectra and time-resolved luminescence signals were measured for $\mathrm{YF}_{3}: \operatorname{Pr}^{3+} 1 \%, \mathrm{Eu}^{3+} x \%$ $(x=0,5$ and 10$)$ as shown in Fig. 1. The experiments show a strong quenching of the $\operatorname{Pr}^{3+1} \mathrm{~S}_{0}$ emission in the presence of $\mathrm{Eu}^{3+}$ ions. This quenching process is attributed to nonradiative relaxation via a $\mathrm{Eu}^{2+}-\mathrm{Pr}^{4+}$ metal-to-metal charge-transfer state. Quenching of the $\operatorname{Pr}^{3+1} \mathrm{~S}_{0}$ emission by $\mathrm{Yb}^{3+}$ confirms this quenching mechanism.

An alternative to transferring the ${ }^{1} \mathrm{~S}_{0^{-}}{ }^{1} \mathrm{I}_{6},{ }^{3} \mathrm{P}_{\mathrm{J}}$ energy to a lanthanide ion $\left(\right.$ like $\mathrm{Eu}^{3+}$ ) is to include a $3 \mathrm{~d}$ transition

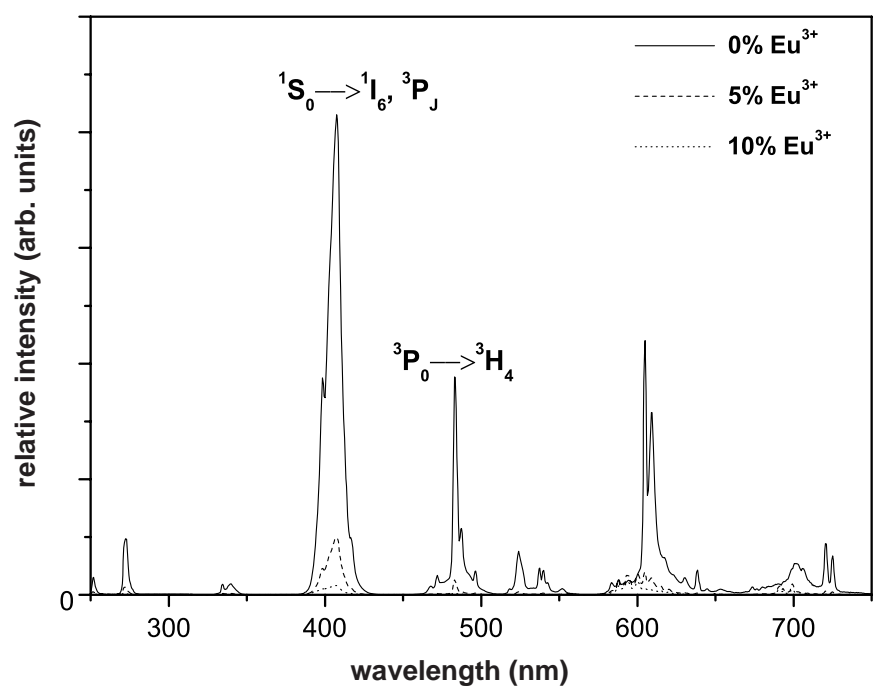

Fig. 1. Emission spectra (RT) of $\mathrm{YF}_{3}$ doped with $1 \% \mathrm{Pr}^{3+}$ and co-doped with $\mathrm{Eu}^{3+}$ plotted on a relative intensity scale. The concentrations of $\mathrm{Eu}^{3+}$ are indicated in the figure. Excitation is into the $\operatorname{Pr}^{3+} 4 \mathrm{f}^{2} \rightarrow 4 \mathrm{f} 5 \mathrm{~d}$ band at $190 \mathrm{~nm}$. The $\mathrm{Pr}^{3+1} \mathrm{~S}_{0} \rightarrow{ }^{1} \mathrm{I}_{6},{ }^{3} \mathrm{P}_{\mathrm{J}}$ and ${ }^{3} \mathrm{P}_{0} \rightarrow{ }^{3} \mathrm{H}_{4}$ transitions are indicated in the figure. Note the quenching of the $\operatorname{Pr}^{3+}$ emissions when $\mathrm{Eu}^{3+}$ is codoped.
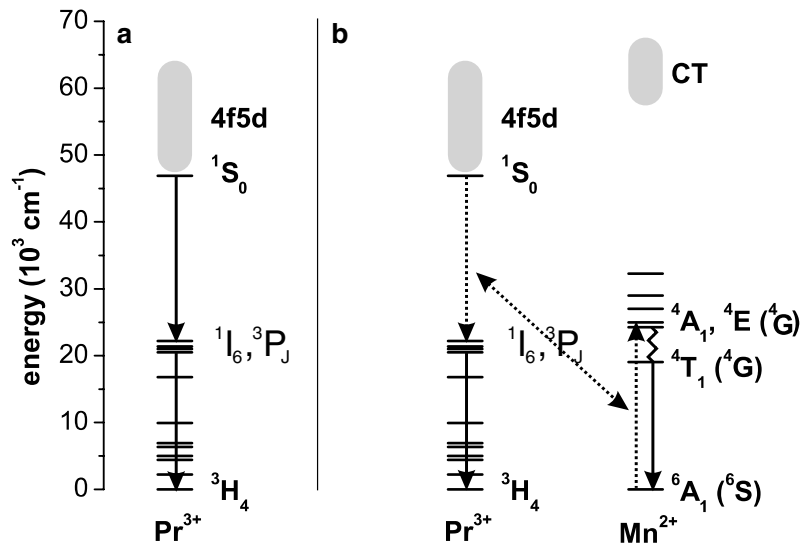

Fig. 2. Schematic of the $\mathrm{Pr}^{3+}$ cascade emission (left hand side) and the desired energy-transfer process between $\operatorname{Pr}^{3+}\left({ }^{1} \mathrm{~S}_{0}\right)$ and $\mathrm{Mn}^{2+}$ (right hand side). Solid lines denote radiative transitions. CT stands for charge transfer. The energy-transfer process is depicted by dotted lines. The zigzag line denotes non-radiative relaxation. After the energy-transfer process both $\operatorname{Pr}^{3+}$ and $\mathrm{Mn}^{2+}$ each emit one photon.

metal ion as an acceptor. In particular, energy transfer to $\mathrm{Mn}^{2+}\left(3 \mathrm{~d}^{5}\right)$ is promising. The $\mathrm{Mn}^{2+}$ ion has narrow absorption bands around $400 \mathrm{~nm}$ due to transitions from the ${ }^{6} \mathrm{~A}_{1}$ ground state to ${ }^{4} \mathrm{~A}_{1}$ and ${ }^{4} \mathrm{E}$ excited states. The cross-relaxation scheme is shown in Fig. 2. In view of the spectral overlap between the ${ }^{1} \mathrm{~S}_{0} \rightarrow{ }^{1} \mathrm{I}_{6},{ }^{3} \mathrm{P}_{\mathrm{J}}$ emission and the $\mathrm{Mn}^{2+}$ excitation lines around $400 \mathrm{~nm}$, energy transfer is expected to occur. To investigate if energy transfer from $\mathrm{Pr}^{3+}$ to $\mathrm{Mn}^{2+}$ does occur, a number of fluoride host lattices in which $\operatorname{Pr}^{3+}$ shows cascade emission (high energy fd state) have been co-doped with $\mathrm{Pr}^{3+}$ and various concentrations of $\mathrm{Mn}^{2+}$. To determine whether or not the desired spectral conversion of the $405 \mathrm{~nm}$ emission occurs by energy transfer to $\mathrm{Mn}^{2+}$, luminescence spectra are recorded and analyzed.

For the downconversion of one VUV photon into two visible photons using $\mathrm{Pr}^{3+}$ and $\mathrm{Mn}^{2+}$ several conditions have to be met: (1) there should be a cascade emission originating from the ${ }^{1} \mathrm{~S}_{0}$ level of the $\operatorname{Pr}^{3+}$ ion (i.e. the lowestenergy fd state has to be higher in energy than the ${ }^{1} \mathrm{~S}_{0}$ state), (2) there should be spectral overlap between the $\mathrm{Pr}^{3+1} \mathrm{~S}_{0} \rightarrow{ }^{1} \mathrm{I}_{6}$ emission lines and absorption lines of the $\mathrm{Mn}^{2+}$ ion in the fluoride host lattice and (3) the distance between the Pr and Mn ions needs to be small to allow for interaction between ions.

Optical spectroscopy experiments on singly doped (Pr or $\mathrm{Mn}$ ) systems provide information to show whether or not the first two conditions are met. If this is the case, measurements on doubly doped systems ( $\mathrm{Pr}$ and $\mathrm{Mn}$ ) can be done to investigate if the interaction between neighboring ions is strong enough to give rise to efficient energy transfer. For all fluoride host lattices investigated in this section, the first two conditions are met. As a typical example, only the results for $\mathrm{SrY}_{2} \mathrm{~F}_{8}$ are discussed in detail.

In Fig. 3 luminescence spectra are shown for $\mathrm{SrY}_{2} \mathrm{~F}_{8}$ samples doped with a single type of ion ( $\operatorname{Pr}$ or $\mathrm{Mn})$ and 

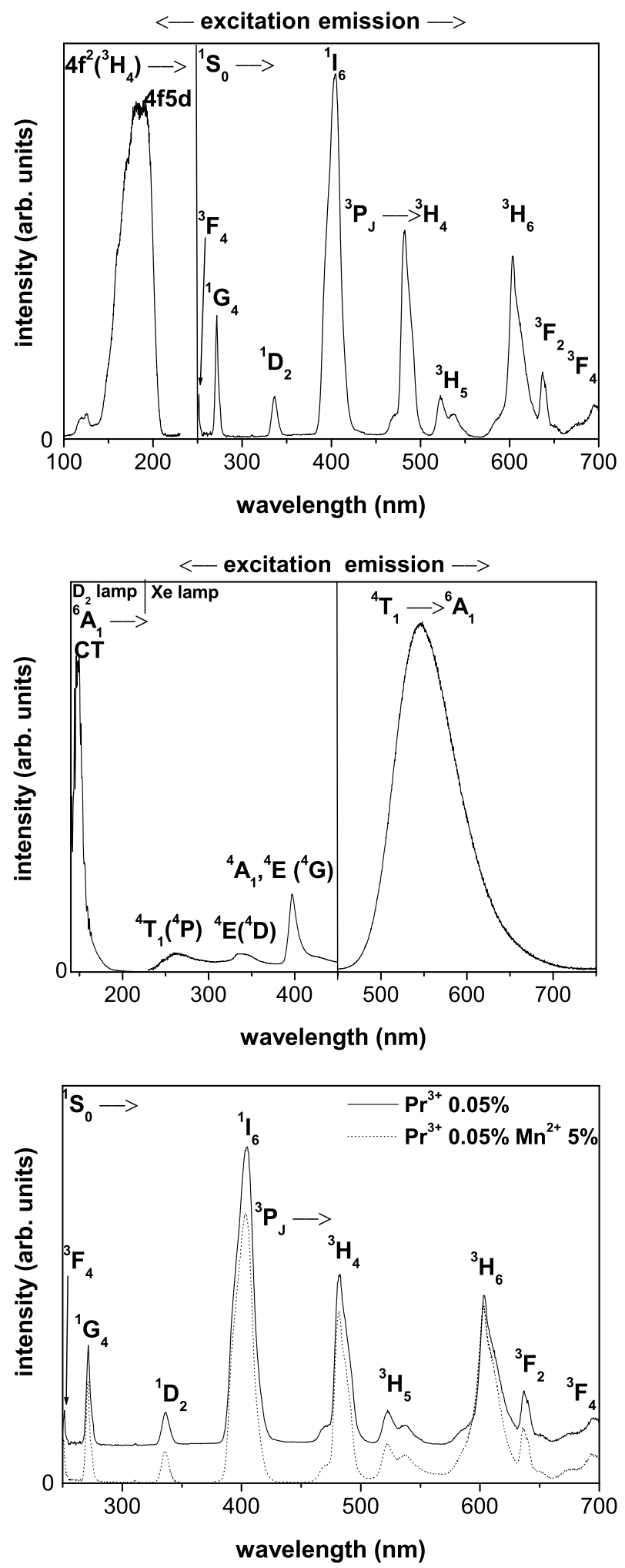

Fig. 3. (top) Excitation (10 K, $\left.\lambda_{m}=401 \mathrm{~nm}\right)$ and emission (RT, $\lambda_{x}=186 \mathrm{~nm}$ ) spectra for $\mathrm{SrY}_{2} \mathrm{~F}_{8}: \mathrm{Pr}^{3+} 0.05 \%$, (middle) Excitation (RT, $\left.\lambda_{m}=555 \mathrm{~nm}\right)$ and emission (RT, $\left.\lambda_{x}=161 \mathrm{~nm}\right)$ spectra for $\mathrm{SrY}_{2} \mathrm{~F}_{8}: \mathrm{Mn}^{2+}$ $1 \%$, (bottom) emission spectra (RT, $\lambda_{x}=185 \mathrm{~nm}$ ) for $\mathrm{SrY}_{2} \mathrm{~F}_{8}$ doped with $0.05 \% \operatorname{Pr}^{3+}$ (drawn line) or with $0.05 \% \operatorname{Pr}^{3+}$ and $5 \% \mathrm{Mn}^{2+}$.

for a doubly doped sample. The emission spectrum of $\mathrm{SrY}_{2} \mathrm{~F}_{8}: \mathrm{Pr}^{3+}$ (Fig. 3(top)) upon excitation in the $4 \mathrm{f} 5 \mathrm{~d}$ band
$(190 \mathrm{~nm})$ shows a number of sharp lines in the UV and visible part of the spectrum. The spectrum is dominated by the strong ${ }^{1} \mathrm{~S}_{0} \rightarrow{ }^{1} \mathrm{I}_{6}$ emission line around $405 \mathrm{~nm}$. In the higher energy region weaker lines corresponding to other ${ }^{1} \mathrm{~S}_{0}$ emissions are observed and in the visible range there are ${ }^{3} \mathrm{P}_{0}$ emission lines. The observation of ${ }^{1} \mathrm{~S}_{0}$ emission from $\operatorname{Pr}^{3+}$ shows that the $4 \mathrm{f} 5 \mathrm{~d}$ state is situated above the ${ }^{1} \mathrm{~S}_{0}$ level. The excitation spectrum of the $\mathrm{Mn}^{2+}$ emission (Fig. 3(middle)) shows a sharp line around $400 \mathrm{~nm}$ and some weaker lines at shorter wavelengths. The $400 \mathrm{~nm}$ line is assigned to the ${ }^{6} \mathrm{~A}_{1 \mathrm{~g}} \rightarrow{ }^{4} \mathrm{~A}_{1 \mathrm{~g}},{ }^{4} \mathrm{E}_{\mathrm{g}}$ transitions within the $3 \mathrm{~d}^{5}$ configuration of $\mathrm{Mn}^{2+}$. The emission spectrum of the $\mathrm{Mn}^{2+}$ ion in $\mathrm{SrY}_{2} \mathrm{~F}_{8}$ consists of a broad band around $560 \mathrm{~nm}$. The luminescence spectra of $\mathrm{SrY}_{2} \mathrm{~F}_{8}: \mathrm{Pr}^{3+}$ and $\mathrm{SrY}_{2} \mathrm{~F}_{8}: \mathrm{Mn}^{2+}$ show that the first two conditions for energy transfer are met: the $4 f 5 \mathrm{~d}$ state is at higher energy than the ${ }^{1} \mathrm{~S}_{0}$ and the ${ }^{1} \mathrm{~S}_{0} \rightarrow{ }^{1} \mathrm{I}_{6}$ emission line of $\mathrm{Pr}^{3+}$ has a good spectral overlap with the ${ }^{6} \mathrm{~A}_{1 \mathrm{~g}} \rightarrow{ }^{4} \mathrm{~A}_{1 \mathrm{~g}},{ }^{4} \mathrm{E}_{\mathrm{g}}$ absorption lines of $\mathrm{Mn}^{2+}$.

To investigate if the energy-transfer process (cross-relaxation) shown in Fig. 2 can occur, the luminescence properties of doubly doped ( $\mathrm{Pr}$ and $\mathrm{Mn}) \quad \mathrm{SrY}_{2} \mathrm{~F}_{8}$ were investigated. In Fig. 3 (bottom) the emission spectra of $\mathrm{SrY}_{2} \mathrm{~F}_{8}: \mathrm{Pr}^{3+}$ are shown for samples with the same $\operatorname{Pr}^{3+}$ concentration $(0.05 \mathrm{~mol} \%)$ and two different $\mathrm{Mn}^{2+}$ concentration $(0 \%$ or $5 \%)$. The emission in the visible spectral range shows that the spectra do not change upon adding $\mathrm{Mn}^{2+}$. If the energy-transfer $\operatorname{Pr}^{3+}\left({ }^{1} \mathrm{~S}_{0} \rightarrow{ }^{1} \mathrm{I}_{6}\right)$ to $\mathrm{Mn}^{2+}\left({ }^{6} \mathrm{~A}_{1 \mathrm{~g}} \rightarrow{ }^{4} \mathrm{~A}_{1 \mathrm{~g}},{ }^{4} \mathrm{E}_{\mathrm{g}}\right)$ occurs, one would expect to observe a $\mathrm{Mn}^{2+}$ emission band around $560 \mathrm{~nm}$ that increases in intensity upon raising the $\mathrm{Mn}$ concentration. The absence of a $\mathrm{Mn}^{2+}$ emission band indicates that energy transfer does not occur. The observation that the Pr emission spectrum does not change is further evidence for the absence of cross-relaxation. Clearly, there is no energy transfer from $\mathrm{Pr}$ and $\mathrm{Mn}$ in spite of the favorable spectral overlap and a considerable number of nearest-neighbor pairs of $\mathrm{Pr}-\mathrm{Mn}$. From this observation one can conclude that even in the nearest-neighbor pairs the energy transfer cannot compete with radiative decay from the ${ }^{1} \mathrm{~S}_{0}$ state. Similar research on $\mathrm{Pr}$ and/or $\mathrm{Mn}$ doped $\mathrm{YF}_{3}, \mathrm{CaF}_{2}$ and $\mathrm{LiBaF}_{3}$ provided similar results: no evidence for energy transfer from $\mathrm{Pr}$ to $\mathrm{Mn}$ is obtained.

The absence of energy transfer is not well-understood. Calculations of the energy-transfer rate for dipole-dipole interaction show that the critical distance for energy transfer is approximately $3.5 \AA$, which is slightly smaller than the nearest-neighbor distance but small enough that some energy transfer and thus $\mathrm{Mn}^{2+}$ emission should be observed.

\section{Cooperative sensitization}

The idea for the two quantum cutting systems discussed in Section 3 is based on resonant energy transfer. Splitting of the energy is achieved by population of an intermediate energy level of the donor. If overlap between donor emis- 
sion and acceptor absorption is absent, second-order downconversion may become the dominant relaxation process (competing with spontaneous emission). In this process a donor excites two acceptors simultaneously. The resonance condition is fulfilled if the sum of the energy of the absorption transitions of the two acceptor centres equals the energy of the emission of the donor.

This cooperative sensitisation process was predicted in 1957 by Dexter [10] but it was only recently that a paper has been published in which the occurrence of cooperative sensitisation (or second-order downconversion) was presented [11]. A promising system to study second-order energy-transfer processes is the combination of one $\mathrm{Tb}^{3+}$ and two $\mathrm{Yb}^{3+}$ ions: the $\mathrm{Tb}^{3+5} \mathrm{D}_{4} \rightarrow{ }^{7} \mathrm{~F}_{6}$ transition is located at approximately twice the energy of the $\mathrm{Yb}^{3+2} \mathrm{~F}_{5 / 2} \rightarrow{ }^{2} \mathrm{~F}_{7 / 2}$ transition and $\mathrm{Yb}^{3+}$ has no other levels up to the UV region (see Fig. 4).

The purpose of this section is to study second-order downconversion in $\mathrm{Tb}-\mathrm{Yb}$ compounds and to elucidate its efficiency and energy-transfer mechanism. Energy transfer in powders of $\mathrm{Yb}_{x} \mathrm{Y}_{(1-x)} \mathrm{PO}_{4}$ doped with $1 \% \mathrm{~Tb}^{3+}$ is studied by emission, excitation and time-resolved luminescence measurements. The time-resolved luminescence measurements are compared with theories for phonon-assisted energy transfer [12] and second-order downconversion through a cooperative or an accretive energy-transfer mechanism [13] by exact calculations and simulations using Monte Carlo methods.

To investigate whether energy transfer from $\mathrm{Tb}^{3+}$ to $\mathrm{Yb}^{3+}$ occurs, an emission spectrum upon excitation in the

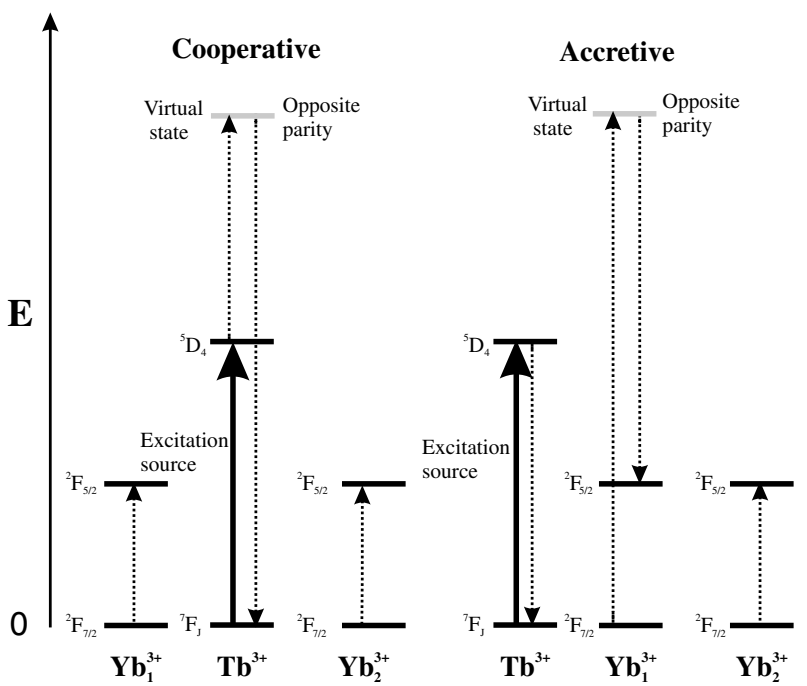

Fig. 4. Schematic representation of the cooperative and accretive pathways for energy transfer from $\mathrm{Tb}^{3+}$ to $\mathrm{Yb}^{3+}$. The bold arrows indicate excitation of $\mathrm{Tb}^{3+}$ into the ${ }^{5} \mathrm{D}_{4}$ state, after which energy transfer may occur. The energy-transfer processes are depicted by the dotted lines. In both mechanisms a virtual state is involved. For the cooperative mechanism, the virtual state is located on $\mathrm{Tb}^{3+}$. For the accretive mechanism, the virtual state is located on $\mathrm{Yb}^{3+}$. Since the total amount of energy is unchanged after the energy transfer, the resonance condition implies that the transition energy of $\mathrm{Tb}^{3+}$ balances the sum of the transition energies of the $\mathrm{Yb}^{3+}$ ions.

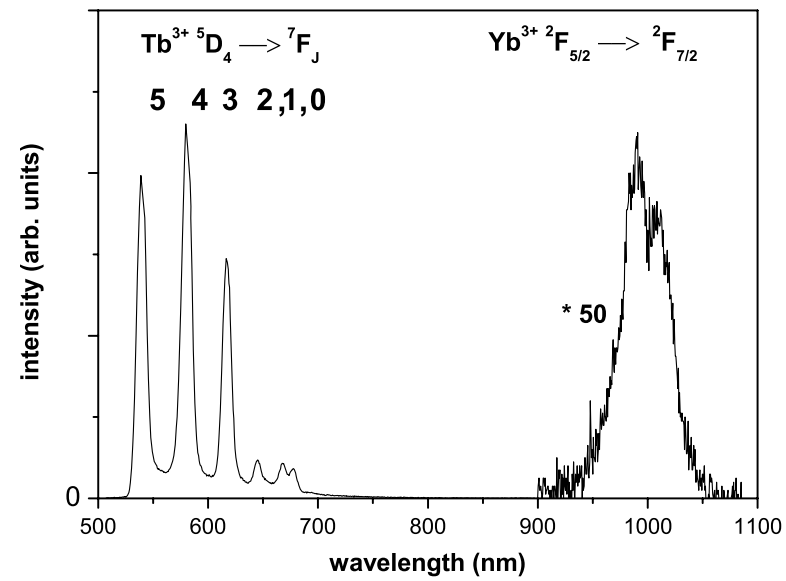

Fig. 5. Visible/near infrared emission spectrum of $\mathrm{Y}_{0.74} \mathrm{Yb}_{0.25} \mathrm{~Tb}_{0.01} \mathrm{PO}_{4}$ upon $\mathrm{Tb}^{3+7} \mathrm{~F}_{6} \rightarrow{ }^{5} \mathrm{D}_{4}$ excitation $(489 \mathrm{~nm})$. The spectral region between 900 and $1100 \mathrm{~nm}$ is amplified by a factor of 50 .

$\mathrm{Tb}^{3+5} \mathrm{D}_{4}$ level was recorded. Fig. 5 shows the emission spectrum of $\mathrm{Yb}_{0.25} \mathrm{Y}_{0.74} \mathrm{~Tb}_{0.01} \mathrm{PO}_{4}$ at room temperature upon $\mathrm{Tb}^{3+7} \mathrm{~F}_{6} \rightarrow{ }^{5} \mathrm{D}_{4}$ excitation. In the region of 500 $700 \mathrm{~nm}$ a series of lines is observed while in the 900$1100 \mathrm{~nm}$ region an emission band of much lower intensity appears (multiplied 50 times in Fig. 5). The lines in the visible part of the spectrum are due to $\mathrm{Tb}^{3+5} \mathrm{D}_{4} \rightarrow{ }^{7} \mathrm{~F}_{\mathrm{J}}$ multiplet emissions. They are similar to previous measurements on $\mathrm{YPO}_{4}$ doped with $\mathrm{Tb}^{3+}$ [14]. Transitions to different ground state terms with $\mathbf{J}$ ranging from 5 to 0 are clearly resolved in the spectrum. The $\mathrm{Tb}^{3+5} \mathrm{D}_{4} \rightarrow{ }^{7} \mathrm{~F}_{6}$ emission line is absent because of the use of a $540 \mathrm{~nm}$ cut-off filter. This filter was used to prevent second-order $\mathrm{Tb}^{3+}$ emissions in the wavelength region of $900-1100 \mathrm{~nm}$, since these emissions would obscure the detection of $\mathrm{Yb}^{3+}$ emission lines. The features in this part of the spectrum are attributed to the $\mathrm{Yb}^{3+2} \mathrm{~F}_{5 / 2} \rightarrow{ }^{2} \mathrm{~F}_{7 / 2}$ transitions. Compared to the $\mathrm{Tb}^{3+}$ emission, the intensity of the $\mathrm{Yb}^{3+}$ luminescence is weak. This is due to the low response of the grating and the $\sim 15$. In addition concentration quenching (the migration of excitation energy over $\mathrm{Yb}^{3+}$ ions to quenching sites) reduces the luminescence quantum yield.

Excitation spectra of $\mathrm{Tb}^{3+}$ and $\mathrm{Yb}^{3+}$ emission in $\mathrm{Yb}_{0.25} \mathrm{Y}_{0.74} \mathrm{~Tb}_{0.01} \mathrm{PO}_{4}$ were recorded to give convincing evidence for the presence of $\mathrm{Tb}^{3+} \rightarrow \mathrm{Yb}^{3+}$ energy transfer. Fig. 6 shows the wavelength region where the $\mathrm{Tb}^{3+7} \mathrm{~F}_{6} \rightarrow{ }^{5} \mathrm{D}_{4}$ transition is located. The solid line depicts the excitation spectrum of the $\mathrm{Tb}^{3+5} \mathrm{D}_{4} \rightarrow{ }^{7} \mathrm{~F}_{3}$ emission. The dotted line shows the excitation spectrum of the $\mathrm{Yb}^{3+2} \mathrm{~F}_{5 / 2} \rightarrow{ }^{2} \mathrm{~F}_{7 / 2}$ emission. In both spectra lines are located around $485-495 \mathrm{~nm}$. For the spectrum monitoring the $\mathrm{Tb}^{3+5} \mathrm{D}_{4} \rightarrow{ }^{7} \mathrm{~F}_{3}$ emission, the excitation lines are ascribed to the well-known ${ }^{7} \mathrm{~F}_{6} \rightarrow{ }^{5} \mathrm{D}_{4}$ transitions. The excitation spectrum of the $\mathrm{Yb}^{3+}$ emission is very similar to the $\mathrm{Tb}^{3+5} \mathrm{D}_{4} \rightarrow{ }^{7} \mathrm{~F}_{3}$ excitation spectrum. The observation of the $\mathrm{Tb}^{3+}{ }^{3} \mathrm{~F}_{6} \rightarrow{ }^{5} \mathrm{D}_{4}$ lines in the excitation spectrum of $\mathrm{Yb}^{3+}$ shows that energy transfer from $\mathrm{Tb}^{3+}$ to $\mathrm{Yb}^{3+}$ is present. 


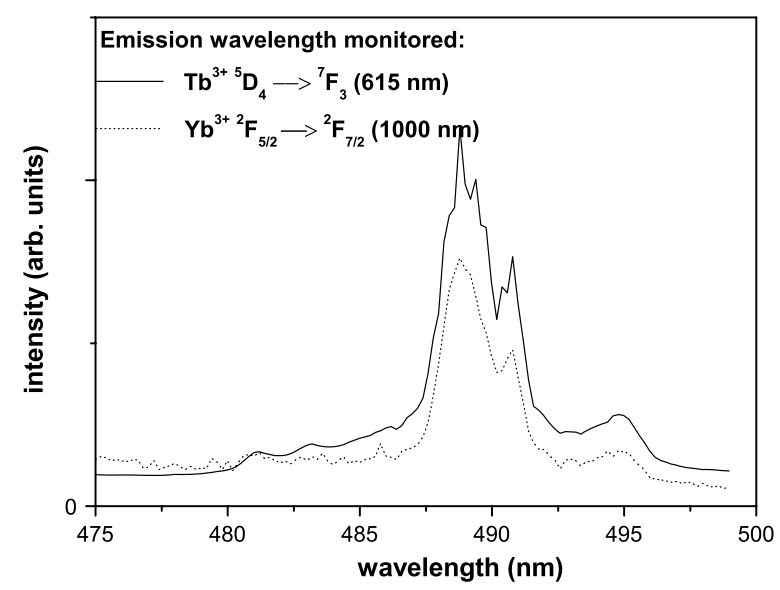

Fig. 6. Excitation spectra of the $\mathrm{Tb}^{3+5} \mathrm{D}_{4} \rightarrow{ }^{7} \mathrm{~F}_{3}$ emission $(615 \mathrm{~nm}$, solid line) and the $\mathrm{Yb}^{3+2} \mathrm{~F}_{5 / 2} \rightarrow{ }^{2} \mathrm{~F}_{7 / 2}$ emission (1000 nm, dotted line) in $\mathrm{Y}_{0.74} \mathrm{Yb}_{0.25} \mathrm{~Tb}_{0.01} \mathrm{PO}_{4}$

The results discussed above provide evidence for the presence of $\mathrm{Tb}^{3+} \rightarrow \mathrm{Yb}^{3+}$ energy transfer. It is interesting to investigate the mechanism and efficiency of the energytransfer process. An alternative for an energy-transfer process involving two $\mathrm{Yb}^{3+}$ ions is mentioned in the literature as phonon-assisted energy transfer $[15,16]$. On the basis of emission and excitation spectra this competing process cannot be excluded. Analysis of the transient luminescence curves provides insight in the energy-transfer mechanism.

In Fig. 7 the decay curves of the $\mathrm{Tb}^{3+5} \mathrm{D}_{4} \rightarrow{ }^{7} \mathrm{~F}_{4}$ luminescence $(544.0 \mathrm{~nm})$ are plotted for $\mathrm{Yb}^{3+}$ concentrations of $0 \%, 5 \%, 15 \%, 25 \%, 50 \%, 75 \%$ and $99 \%$. The $\mathrm{Tb}^{3+}$ emission in $\mathrm{YPO}_{4}: \mathrm{Tb}^{3+} 1 \%$ shows a nearly single exponential decay as expected (only radiative decay). A mono-exponential fit yields a decay time of $2.3 \mathrm{~ms}$. A decay time between 2 and $3 \mathrm{~ms}$ is a typical value compared to $\mathrm{Tb}^{3+5} \mathrm{D}_{4}$ emission in oxides where the site of $\mathrm{Tb}^{3+}$ lacks inversion symmetry [16]. When the $\mathrm{Yb}^{3+}$ concentration is increased, the decay curve decreases more rapidly and it becomes non-exponential. For $\mathrm{YbPO}_{4}: \mathrm{Tb}^{3+} 1 \%$ the fastest decay is observed and the curve is again nearly single exponential. The faster decline as a function of $\mathrm{Yb}^{3+}$ concentration may be explained by the introduction of extra decay pathways due to the $\mathrm{Yb}^{3+}$-doping: energy transfer from $\mathrm{Tb}^{3+}$ ${ }^{5} \mathrm{D}_{4}$ to $\mathrm{Yb}^{3+}$ enhances the $\mathrm{Tb}^{3+}{ }^{5} \mathrm{D}_{4}$ decay rate.

The presence of $\mathrm{Yb}^{3+}$ ions also explains the non-exponential behaviour at intermediate doping concentrations and the single exponential behaviour for $99 \% \mathrm{Yb}^{3+}$. At intermediate doping concentrations the $\mathrm{Yb}^{3+}$ and the $\mathrm{Y}^{3+}$ ions are randomly distributed over the $\mathrm{RE}^{3+}$ lattice sites. Thus, the environment of every $\mathrm{Tb}^{3+}$ ion is different, leading to a variety of transfer rates. At an $\mathrm{Yb}^{3+}$ fraction of $99 \%$ the $\mathrm{Tb}^{3+}$ ions are surrounded by $\mathrm{Yb}^{3+}$ ions only, leading to a single decay component. Therefore, the $99 \% \mathrm{Yb}^{3+}$ decay curve in Fig. 7 is again nearly single exponential. Fitting this curve with a single exponential function yields a decay time of $0.25 \mathrm{~ms}$.

Assuming that the radiative rate of the $\mathrm{Tb}^{3+5} \mathrm{D}_{4}$ emission does not change when $\mathrm{Y}^{3+}$ is substituted for $\mathrm{Yb}^{3+}$,

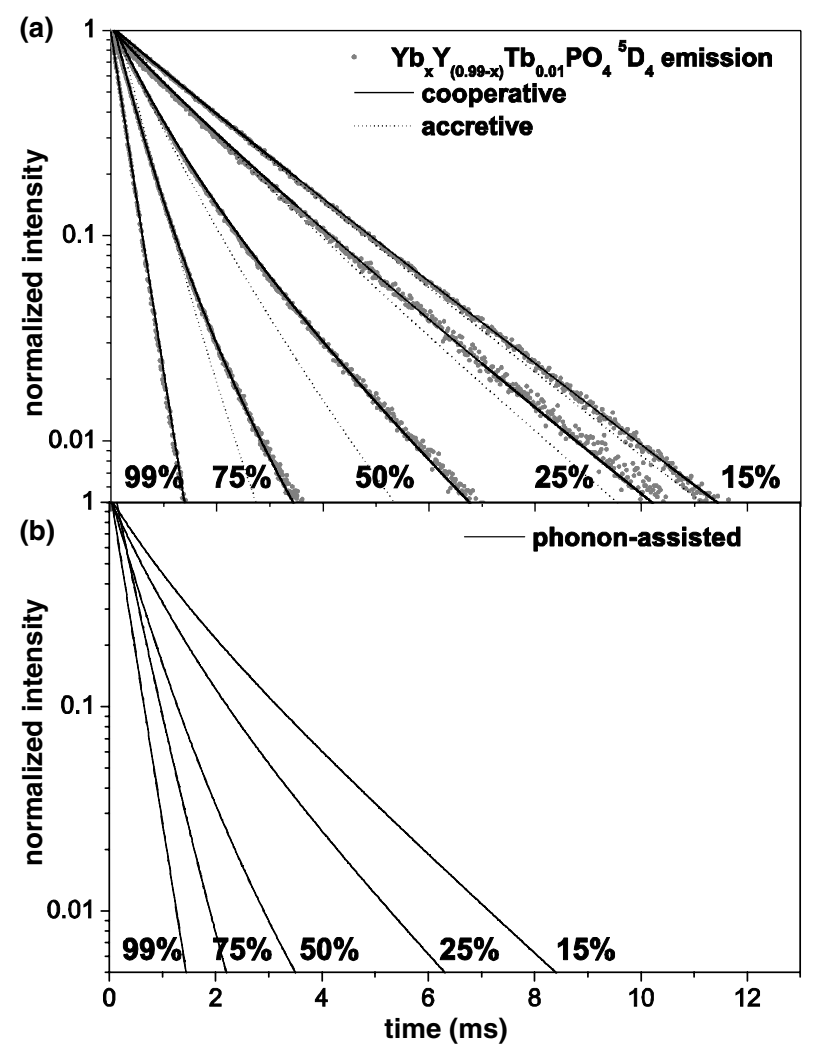

Fig. 7. (a) Luminescence decay curves of the $\mathrm{Tb}^{3+5} \mathrm{D}_{4}$ emission for various concentrations of $\mathrm{Yb}^{3+}$ plotted with simulation results. The dots are the experimental results. The solid lines are simulated curves using a cooperative dipole-dipole model. Dashed lines are simulated curves using an accretive dipole-dipole model (see Fig. 4). (b) Simulated decay curves for the phonon-assisted dipole-dipole model. For both figures the $\mathrm{Yb}^{3+}$ concentrations are indicated in the figure.

the energy-transfer rate in $\mathrm{YbPO}_{4}$ can be calculated by subtracting the $\mathrm{Tb}^{3+5} \mathrm{D}_{4}$ radiative decay rate from the decay rate in $\mathrm{YbPO}_{4}$. This results in an energy-transfer rate of $35 \times 10^{2} \mathrm{~s}^{-1}$ in $\mathrm{YbPO}_{4}: \mathrm{Tb}^{3+} 1 \%$. This is roughly an order of magnitude faster than the radiative decay rate of $\mathrm{Tb}^{3+}$ and indicates that the energy-transfer process is efficient.

To distinguish between the three energy-transfer mechanisms the decay curves were compared to the decay behaviour predicted by Monte Carlo simulations. Experiments and simulated results for the cooperative, the accretive and the phonon-assisted dipole-dipole models are shown in Fig. 7 (see Ref. [4] for further details). To obtain the computed curves, the radiative decay rate and the energytransfer rate in $\mathrm{YbPO}_{4}$ (as determined from single exponential fits to the decay curves of $\mathrm{YPO}_{4}$ and $\mathrm{YbPO}_{4}$ in Fig. 7) were used as input parameters. Therefore, at $99 \%$ $\mathrm{Yb}^{3+}$ concentration, the three simulated curves all match the experimental curve. However, the cooperative model matches the experiments for all $\mathrm{Yb}^{3+}$ concentrations while the accretive and phonon-assisted energy-transfer model deviate substantially. At all intermediate $\mathrm{Yb}^{3+}$ concentrations, the decay profiles of the phonon-assisted model and the accretive model fall off faster than the cooperative model. The excellent agreement between the experimentally 
measured luminescence decay curves and the calculated curves for cooperative energy transfer via dipole-dipole interaction provides strong evidence that this mechanism is operative in the $(\mathrm{Y}, \mathrm{Yb}) \mathrm{PO}_{4}: \mathrm{Tb}^{3+}$ system.

The energy-transfer efficiency is shown to be $88 \%$ for $\mathrm{YbPO}_{4}: \mathrm{Tb}^{3+} 1 \%$. This means that 88 out of $100 \mathrm{~Tb}^{3+}$ ions each transfer their energy to two $\mathrm{Yb}^{3+}$ ions. This makes a quantum efficiency of $188 \%$ possible. Materials exploiting $\mathrm{Tb}-\mathrm{Yb}$ cooperative quantum cutting may be useful to increase the efficiency of silicon-based solar cells by downconverting the green-to-UV part of the solar spectrum to $\sim 1000 \mathrm{~nm}$ photons, with almost complete doubling of the number of photons. The weak IR emission of the presently studied materials (see Fig. 5) shows that, although the physics of the energy-transfer process allow for efficient quantum cutting, the actual quantum efficiency in the IR is still low. In order to obtain a material with an actual quantum efficiency close to $200 \%$, two drawbacks should be overcome. First, due to the high $\mathrm{Yb}^{3+}$ concentration needed for efficient downconversion, concentration quenching of the $\mathrm{Yb}^{3+}$ emission is a major issue. To overcome this problem, incorporation of ions with an excited state at slightly lower energy than the ${ }^{2} \mathrm{~F}_{5 / 2}$ state of $\mathrm{Yb}^{3+}$ ion (but still higher than the bandgap of silicon) may be used. These ions will function as traps for the energy in the ${ }^{2} \mathrm{~F}_{5 / 2}$ state so that migration of excitation energy to quenching sites is suppressed. Another possibility is the use of one-dimensional systems in order to localize the energy migration. The second obstacle is the low absorption cross section for green-to-UV light of $\mathrm{Yb} / \mathrm{YPO}_{4}$ doped with $\mathrm{Tb}^{3+}$. The absorption cross section may be enhanced in two ways. First, a bulk material with a lower bandgap may be used so that the host itself would absorb part of the solar spectrum, after which energy transfer to a $\mathrm{Tb}^{3+}$ ion should occur. Second, sensitizer ions could be used to absorb the desired part of the solar spectrum and transfer the excitation energy to the ${ }^{5} \mathrm{D}_{4}$ state of $\mathrm{Tb}^{3+}$ for the $\mathrm{Tb}$ $\mathrm{Yb}$ downconversion process.

\section{Conclusion}

The possibility of efficient visible quantum cutting has been investigated for materials in which $\operatorname{Pr}^{3+}$ shows a cascade emission from the ${ }^{1} \mathrm{~S}_{0}$ level. In order to convert the $405 \mathrm{~nm}$ photon emitted by $\mathrm{Pr}^{3+}$ in the first step into a more useful visible wavelength, two types of co-activators were added, viz. $\mathrm{Eu}^{3+}$ or $\mathrm{Mn}^{2+}$. In the case of $\mathrm{Eu}^{3+}$ a quenching of the ${ }^{1} \mathrm{~S}_{0}$ emission from $\operatorname{Pr}^{3+}$ is observed. The quenching is ascribed to relaxation through a metal-to-metal chargetransfer state. Upon adding $\mathrm{Mn}^{2+}$ as a co-activator, no energy transfer is observed even at concentrations as high as $5 \%$. The origin of the absence of energy transfer is unclear. In the second part of the paper second-order downconversion in $\mathrm{Yb}_{x} \mathrm{Y}_{(1-x)} \mathrm{PO}_{4}: \mathrm{Tb}^{3+} 1 \%$ is studied. Emission, excitation, and time-resolved luminescence measurements reveal the occurrence of energy transfer from the ${ }^{5} \mathrm{D}_{4}$ level of $\mathrm{Tb}^{3+}$ to two $\mathrm{Yb}^{3+}$ ions. Monte Carlo simulations of the luminescence decay curves are shown to be a useful tool for the determination of the energy-transfer mechanism. When a cooperative dipole-dipole model is assumed, the simulated and the experimental curves show an excellent agreement for all $\mathrm{Yb}^{3+}$ concentrations. Based on the poor agreement between simulated and measured luminescence decay curves, it is possible to exclude an accretive dipole-dipole mechanism and a phonon-assisted energy-transfer process. The efficiency of the quantum cutting process is $88 \%$ in $\mathrm{YbPO}_{4}: \mathrm{Tb}^{3+} 1 \%$. This allows for an overall quantum efficiency (VIS + IR) of $188 \%$, which is close to the limit of $200 \%$.

\section{Acknowledgements}

The authors are grateful to H.W. de Wijn and M. Giesselbach for helpful discussions. The work described here was supported by the Council for Chemical Sciences $(\mathrm{CW})$, with financial aid from the Netherlands Foundation for Technical Research (STW).

\section{References}

[1] G. Blasse, B.C. Grabmaier, Luminescent Materials, Springer Verlag, Berlin, 1994.

[2] C. Ronda, J. Alloys, Compounds 225 (1995) 534.

[3] R.T. Wegh, H. Donker, K.D. Oskam, A. Meijerink, Science 283 (1999) 663.

[4] P. Vergeer, T.J.H. Vlugt, M.H.F. Kox, M.I. den Hertog, J.P.J.M. van der Eerden, A. Meijerink, Phys. Rev. B 71 (2005) 014119.

[5] P.S. Peijzel, A. Meijerink, R.T. Wegh, M.F. Reid, G.W. Burdick, J. Solid State Chem. 178 (2) (2005) 448.

[6] L. van Pieterson, M.F. Reid, R.T. Wegh, S. Soverna, A. Meijerink, Phys. Rev. B 65 (2002) 045113.

[7] W.W. Piper, J.A. DeLuca, F.S. Ham, J. Lumin. 8 (1974) 344.

[8] J.L. Sommerdijk, A. Bril, A.W. de Jager, J. Lumin. 9 (1974) 288.

[9] M. Zachau, F. Zwaschka, F. Kummer, Proc. Electrochem. Soc. 97 (29) (1998) 314.

[10] D.L. Dexter, Phys. Rev. 108 (1957) 630.

[11] T.T. Basiev, M.E. Doroshenko, V.V. Osiko, JETP Lett. 71 (2000) 8.

[12] T. Miyakawa, D.L. Dexter, Phys. Rev. B 1 (1970) 2961.

[13] D.L. Andrews, R.D. Jenkins, J. Chem. Phys. 114 (2001) 1089.

[14] R.C. Ropp, J. Electrochem. Soc. 115 (1968) 841.

[15] W. Strek, P. Deren, A. Bednarkiewicz, J. Lumin. 87 (9) (2000) 999.

[16] I.R. Martin, A.C. Yanes, J. Mendez-Ramos, M.E. Torres, V.D. Rodriguez, J. Appl. Phys. 89 (2001) 2520. 